\title{
NÍVEL DE ATIVIDADE FÍSICA E ESTADO NUTRICIONAL DE POLICIAIS MILITARES NA CIDADE DE FLORIANO-PI.
}

DOI: 105902/0102830818888

Data de envio: 22/07/2015

Data de aceite: $28 / 10 / 2015$

\section{Eurides Soares De Araújo Reis Neta}

Universidade Estadual do Piauí - UESPI

Faculdade Santo Agostinho - FSA

euridesaraujo_2009@hotmail.com

José Fernandes Filho

Universidade Federal do Rio de Janeiro - UFRJ

centrodeexcelencia@gmail.com

Antonio Carlos Leal Cortez

Faculdade Santo Agostinho - FSA

antoniocarloscortez@hotmail.com

RESUMO: Objetivou-se avaliar o nível de atividade física e o estado nutricional dos policiais militares da cidade de Floriano-Piauí. A pesquisa foi realizada no $3^{\circ}$ Batalhão de Policia Militar da cidade de Floriano, sempre às 6:30h da manhã para as avaliações antropométricas e para avaliação do Nível da Atividade Física utilizou-se o questionário IPAQ. De acordo com os resultados apresentados, pode-se concluir que, os policiais militares do $3^{\circ}$ Batalhão da cidade de Floriano estão fora da normalidade populacional no que se refere ao \%G, RCQ, IMC e inativos segundo o IPAQ, apresentando alto ou moderado risco a saúde.

Palavras-chave: Estado Nutricional. Nível de atividade Física. Policial Militar. 


\section{INTRODUÇÃO}

Segundo Matsudo e Matsudo (2000), atividade física é qualquer movimento corporal que conseguimos realizar em nosso cotidiano, em função de contração muscular e com gasto energético acima do basal. Dessa forma, podemos dividir em dois grandes grupos: estruturada e não estruturada. A não estruturada esta relacionada às atividades da vida diária que envolve gasto de energia, mais que não tem planejamento ou objetivo definido. Enquanto a estruturada caracteriza-se por um programa sistematizado de exercício físico, com planejamento e metas definidas, orientado por um profissional de educação física e esta pode ter inúmeros objetivos, mas a principal meta de qualquer programa de condicionamento físico deve ser a melhora da saúde (ARENA, 2009).

Assim, concorda-se com Porto (2003) quando este recomenda que todo ser humano deve manter a preocupação de promover e manter a saúde, sendo ressaltada para população mundial que, cada vez mais, é necessário à rotina diária a prática de exercício físico regular para combater os efeitos nocivos da vida sedentária. Segundo Sávio et al., (2008) doenças geradas pela hipocinesia que acometem maior parte da população como, diabetes tipo 2, hipertensão arterial, obesidade mórbida, arteriosclerose múltipla, osteoporose dentre outras, podem ser prevenidas com a prática regular de exercícios físicos que também contribuem para o melhor condicionamento físico, o que resulta em uma melhor qualidade de vida em todos os aspectos.

Para aproveitar as vantagens da atividade física, torna-se suficiente aumentar o grau de integração de vida diária à atividade física, combatendo o sedentarismo e seus riscos para a saúde. De acordo com a Organização Mundial de Saúde (2002), e Martins (2000), relatam que a atividade física oferece benefícios na aparência, no trabalho, na saúde, no dia-a-dia, e nos aspectos psicológicos do ser humano.

Segundo Lopes (2008) no Brasil, cerca de 13\% dos indivíduos adultos praticam atividade física de lazer com alguma regularidade, sendo que a prática 
do exercício físico é maior entre os homens. Dessa forma faz-se importante reiterar, os benefícios gerados pela adoção de um estilo de vida, referentes à prática regular de atividade física, boa alimentação, hábitos saudáveis de vida, que podem contribuir de forma efetiva na redução do surgimento das doenças crônico-degenerativas. Ressalta-se ainda que a atividade física regular ajuda na manutenção e/ou perda do peso corporal pelo aumentando do metabolismo. Diante disso, o objetivo desse estudo foi identificar o nível de atividade física dos policiais militares na cidade de Floriano-Piauí.

\section{METODOLOGIA}

\section{Tipo de Estudo}

A referida pesquisa trata-se de um estudo descritivo, quantitativo e de campo.

\section{População e Amostra}

A população da pesquisa foi formada por 199 policiais militares da cidade de Floriano-PI de ambos os sexos, destes foi escolhido uma amostra de 51 policiais do sexo masculino.

\section{Critérios de Inclusão}

Para a amostra foram adotados como critérios de inclusão na pesquisa ter aceitado a participar da pesquisa assinando o termo de TCLE (Termo de Consentimento Livre e Esclarecido), ter entre 20 a 55 anos de idade, ter no mínimo 5 anos de atividade laboral na policia militar e ser devidamente registrado na mesma, e estar presente no local e na hora marcada para a aplicação dos questionários e as avaliações antropométricas neste último estando devidamente caracterizado para tal. 


\section{Critérios de Exclusão}

Para a exclusão da amostra foram usados os critérios de não aceitar assinar o termo de TCLE (Termo de Consentimento Livre e Esclarecido), não está presente nos dias estabelecidos para coleta de dados, ter no mínimo 5 anos de serviço, ser registrado como PM e ter idade abaixo de 20 anos e acima de 55.

\section{Local de coleta de dados}

A pesquisa foi realizada no $3^{\circ}$ Batalhão de Policia Militar da cidade de FlorianoPI, no mês de maio de 2013, sendo solicitado a todos, que chegassem ao local de pesquisa, às 6:30h da manhã para as avaliações antropométricas.

\section{Instrumentos de coleta de dados}

A referida pesquisa teve como instrumentos de coleta dados um questionário estruturado, que avalia o nível de atividade física do sujeito avaliado denominado de IPAQ (Questionário Internacional de Atividade Física). Já para as avaliações antropométricas foram utilizados o peso, a estatura, Índice de massa corporal (IMC), RCCQ (Relação Circunferência Cintura-Quadril) e o Somatório de Dobras Cutâneas para os homens.

\section{Nível de Atividade Física}

Para analisar os dados do nível de atividade física foi usado o consenso realizado entre o Centro de Estudos do Laboratório de Aptidão Física de São Caetano do Sul (CELAFISCS) e o Center for Disease Control (CDC) de Atlanta em 2002 considerando os critérios de freqüência e duração, que classifica as pessoas em cinco categorias:

1. MUITO ATIVO: aquele que cumpriu as recomendações de: a) VIGOROSA: $\geq 5$ dias/sem e $\geq 30$ minutos por sessão e/ou b) VIGOROSA: $\geq 3$ dias/sem e $\geq$ 20 minutos por sessão + MODERADA e/ou CAMINHADA: $\geq 5$ dias/sem e $\geq 30$ 
minutos por sessão.

2. ATIVO: aquele que cumpriu as recomendações de: a) VIGOROSA: $\geq 3$ dias/ sem e $\geq 20$ minutos por sessão; e/ou b) MODERADA ou CAMINHADA: $\geq 5$ dias/ sem e $\geq 30$ minutos por sessão; e/ou c). Qualquer atividade somada: $\geq 5$ dias/ sem e $\geq 150$ minutos/sem (caminhada + moderada + vigorosa).

3. IRREGULARMENTE ATIVO: aquele que realiza atividade física, porém insuficiente para ser classificado como ativo, pois não cumpre as recomendações quanto à frequência ou duração. Para realizar essa classificação somasse a frequência e a duração dos diferentes tipos de atividades (caminhada + moderada + vigorosa). Este grupo foi dividido em dois subgrupos de acordo com o cumprimento ou não de alguns dos critérios de recomendação.

IRREGULARMENTE ATIVO A: aquele que atinge pelo menos um dos critérios da recomendação quanto à frequência ou quanto à duração da atividade: a) Frequência: 5 dias /semana ou b) Duração: 150 min / semana IRREGULARMENTE ATIVO B: aquele que não atingiu nenhum dos critérios da recomendação quanto à frequência nem quanto à duração.

4. SEDENTÁRIO: aquele que não realizou nenhuma atividade física por pelo menos 10 minutos contínuos durante a semana.

\section{Avaliação Antropométrica}

Peso

Para mensura o peso corporal foi utilizado uma balança digital da marca Camry com capacidade $150 \mathrm{Kg}$ e divisão em $100 \mathrm{~g}$. Para a aferição foi seguindo o protocolo que segundo Pitanga (2008, p. 89) cada avaliado com mínimo de roupa possível (com short) e sem calçados, subiu na balança e de costas para escala de medida e aferido o peso. 


\section{Estatura}

Para aferição da estatura segundo Fontoura; Formentin; Abech (2008) foi utilizado uma fita métrica da marca Cescorf (com resolução de $2 \mathrm{~m}$ ), fixada na parede sendo que cada avaliado ao encostar na parede ficou na posição ortostática (em pé), com os pés unidos e descalços, em apnéia inspiratória, de modo a minimizar possíveis variações sobre a variável antropométrica, a cabeça orientada no plano de Frankfurt, paralela ao solo, foi posto uma régua sobre a cabeça do avaliado para aferição da medida.

\section{Índice de massa corporal}

Após a mensuração do peso e da estatura o IMC é calculado com a divisão da massa corporal (peso corporal em kg) pela estatura (estatura em metros) elevada ao quadrado (JÁEQUIER, 1987).

Fórmula: IMC= PESO $(\mathrm{Kg}) / \operatorname{ESTATURA}\left(\mathrm{m}^{2}\right)$.

\begin{tabular}{|l|l|}
\hline IMC (Kg/m 2) & Classificação \\
\hline$<16,0$ & Magreza grau III \\
\hline $16,0-16,9$ & Magreza grau II \\
\hline $17,0-18,4$ & Magreza grau I \\
\hline $18,5-24,9$ & Eutrofia \\
\hline $25,0-29,9$ & Pré-obeso \\
\hline $30,0-34,9$ & Obesidade grau I \\
\hline $35,0-39,9$ & Obesidade grau II \\
\hline$\geq 40,0$ & Obesidade grau III \\
\hline
\end{tabular}

Quadro 1: Classificação do estado nutricional segundo pontos de corte do IMC segundo OMS 1995 e 1997.

\section{Dobras cutâneas}

Para mensurar as medidas de dobras cutâneas foi utilizado um adipômetro da marca Innovare Cescorf de acordo com o protocolo de Jackson, Pollock e Ward (1980), marcou-se com uma caneta as dobras cutâneas tríceps, supra-ilíacas e 
abdominal, depois com a mão esquerda com os dedos indicador e polegar em forma de pinça cerca de um centímetro do ponto de reparo, com a dobra segura, colocou-se o adipômetro, esperando cerca de dois a três segundos para leitura da dobra.

Logo após todas essas medidas de dobras cutâneas foram feitos cálculos de acordo com a equação de GUEDES (1985).

$$
\Sigma=1,17136-0,06706 \text { * LOG10 (Dobra cutânea tricipital + Dobra cutânea }
$$
supra-iliaca + Dobra cutânea abdominal).

\begin{tabular}{|l|c|}
\hline ABAIXO DO NORMAL & ATÉ $12 \%$ \\
\hline NORMAL & 12 a $18 \%$ \\
\hline ACIMA DO NORMAL & 18 a $25 \%$ \\
\hline TENDÊNCIA À OBESIDADE & $>25 \%$ \\
\hline
\end{tabular}

Quadro 2: Classificação do percentual de gordura corporal - masculino segundo pontos de corte do somatório de dobras cutâneas segundo Pitanga (2008).

\section{Circunferências}

Para realização das medidas de RCCQ (Relação Circunferência CinturaQuadril), foi utilizado o protocolo de Callaway et al. (1988) apud Pitanga (2008) como referência seguindo a padronização, as medidas de circunferências foram realizadas na cintura, e no quadril, sendo que, para mensuração da cintura os sujeitos permaneceram na posição ortostática, com o abdômen relaxado, a fita foi posicionada no plano horizontal, ao nível natural da cintura, que é a parte mais estreita do tronco. Para as medidas do quadril, os sujeitos continuaram na posição ortostática, sendo a fita posicionada no plano horizontal, ao nível do ponto de maior circunferência da região glútea. Utilizando uma fita métrica da marca Cescorf (com resolução de $2 \mathrm{~m}$ ). 


\begin{tabular}{|c|c|c|c|c|}
\hline IDADE & BAIXO & MODERADO & ALTO & MUITO ALTO \\
\hline Até 29 & $<0,83$ & $0,83-0,88$ & $0,89-0,94$ & $>0,94$ \\
\hline $30-39$ & $<0,84$ & $0,84-0,91$ & $0,92-0,96$ & $>0,96$ \\
\hline $40-49$ & $<0,88$ & $0,88-0,95$ & $0,96-1,00$ & $>1,00$ \\
\hline $50-59$ & $<0,90$ & $0,90-0,96$ & $0,97-1,02$ & $>1,02$ \\
\hline$>59$ & $<0,91$ & $0,91-0,98$ & $0,99-1,03$ & $>1,03$ \\
\hline
\end{tabular}

Quadro 3: Classificação do RCCQ (Relação Circunferência Cintura-Quadril) - Homens segundo pontos de corte de acordo com Pitanga (2008).

\section{Tabulação dos dados}

Após a coleta dos dados, os mesmos foram tabulados utilizando o programa Microsoft Excel 2007 e analisados estatisticamente, através de frequências simples utilizando o programa Statistical Package for Social Science (SPSS) versão 10.0.

\section{Aspectos éticos}

Foram respeitados os aspectos éticos envolvidos em atividades de pesquisa, que envolvam seres humanos, regulamentados pelas Diretrizes e Normas de Pesquisa em Seres Humanos, através da Resolução 466/12 do Conselho Nacional de Saúde. Para preservar os aspectos éticos e legais dessa pesquisa, um termo de consentimento foi assinado por todos os sujeitos da pesquisa, garantindo aos mesmos anonimatos.

\section{RESULTADOS E DISCUSSÃO}

A amostra deste estudo foi constituída por 51 policiais militares do sexo masculino, com idade variando entre 34 a 53 anos com idade média de 46,39ะ4,25.

Na tabela 1 são apresentadas as variáveis que caracterizam a amostra, além da informação sobre o tempo de efetivo serviço dos policiais militares. 


\begin{tabular}{|c|c|c|c|c|}
\hline Variáveis & Mínimo & Máximo & Média & Desvio Padrão \\
\hline Idade & 34 & 53 & 46,39 & 4,252 \\
\hline Tempo de Trabalho em anos & 18 & 32 & 26,74 & 2,338 \\
\hline Peso & 58 & 110 & 77,80 & 12,228 \\
\hline IMC & 20 & 35 & 27,39 & 3,449 \\
\hline$\% \mathrm{G}$ & 15 & 33 & 27,35 & 3,213 \\
\hline
\end{tabular}

Tabela 1. Características gerais dos policiais militares do $3^{\circ}$ Batalhão da cidade de Floriano-PI

Na tabela 2 são apresentados dos dados referentes a distribuição dos policiais militares segundo a classificação do índice de massa corporal (IMC). De acordo com a Tabela 2, 26 policiais militares estão pré-obesos totalizando $51 \%$ da amostra. Estudo realizado por Reis (2009) com policiais militares do $22^{\circ}$ batalhão do estado de Goiás, identificou $52 \%$ da amostra com sobrepeso. Já em relação ao estudo realizado por Minayo; Assis; Oliveira, (2011) cerca de 48,3\% dos policiais militares do Rio de Janeiro foram classificados com sobrepeso. Tendo em resultados encontrados, observa-se que os policiais militares do $3^{\circ}$ Batalhão da cidade de Floriano, de acordo com média obtida, estão dentro da classificação de pré-obesos, fato preocupante, uma vez que esse quadro conduz a uma série de agravos a saúde para qualquer pessoa, mormente aos policiais militares que tem seu corpo como ferramenta de trabalho.

O grande número de Policiais Militares na faixa de pré-obesidade requer medidas urgentes a serem tomadas pela instituição, adotando políticas que possibilitem aos Policiais Militares, à possibilidade de ter acesso à prática orientada e regular de exercícios físicos, visando à manutenção e a melhoria do seu condicionamento físico e por conseqüência de uma composição corporal satisfatória ao desempenho de suas atividades.

Os valores médios obtidos podem ser considerados altos e de relevância para posterior tomada de decisões visando minimizar este quadro insatisfatório de saúde, pois a obesidade é uma doença que conduz a uma série de outros 
agravos a saúde indesejável para qualquer pessoa, mormente para um Policial que tem no seu corpo a sua principal ferramenta de trabalho.

\begin{tabular}{ccc} 
Classificação IMC & $\mathrm{n}$ & $\%$ \\
\hline Eutrofia & 13 & 25,5 \\
Pré-Obeso & 26 & 51,0 \\
Obesidade Grau I & 11 & 21,6 \\
Obesidade Grau II & 1 & 2,0 \\
Total & 51 & 100 \\
\hline \hline
\end{tabular}

Tabela 2. Distribuição dos policiais militares segundo a classificação do índice de massa corporal (IMC), de acordo com a OMS 1995 e 1997. Floriano-PI.

A tabela 3 apresenta os resultados referentes a distribuição dos policiais militares em relação ao risco de doenças coronarianas, tomando como base a classificação da RCQ. Segundo a Tabela 3 a amostra possui uma taxa de 54,9\% (28) dos policiais militares com risco moderado de doenças coronarianas. Dados que podem ser corroborados de acordo com levantamento realizado por Luz; Lucas; Caputo, (2011) com 14 policiais militares (Sargentos, Cabos e Soldados) do sexo masculino do Estado de Santa Catarina apresentando-se com risco moderado.

\begin{tabular}{ccc}
\hline \hline & & \\
Classificação RCQ & $\mathrm{n}$ & $\%$ \\
\hline Baixo Risco & 8 & 15,7 \\
Risco Moderado & 28 & 54,9 \\
Alto Risco & 10 & 19,6 \\
Risco Muito Alto & 5 & 9,8 \\
Total & 51 & 100 \\
\hline \hline
\end{tabular}

Tabela 3. Distribuição dos policiais militares em relação ao risco de doenças coronarianas segundo classificação do RCQ, de acordo Bray e Grayt (1988); Heyward e Stolarczyk (1996) apud Pitanga (2008). Floriano-PI 
Na tabela 4, são apresentados os resultados indicativos a distribuição dos policiais militares segundo a classificação da \% Gordura. Os valores estimados de G\%, através da densidade corporal são apresentados na Tabela 4, que segundo a classificação de Pitanga (2008) 82,4\% dos policiais militares estão com tendência à obesidade. Em estudo realizado por Luz; Lucas; Caputo, (2011) com o Grupo COBRA do Batalhão de Operações Policiais Especiais do Estado de Santa Catarina no ano de 2010 , a média de $\mathrm{G} \%$ foi de $16,1 \pm 4,4 \%$ estimado a parti da equação de Petroski (1999). Nobre (2008) realizou um levantamento antropométrico com bombeiros e policiais militares da região do Cariri Cearense (idade média 35,8 $\pm 4,9$ anos) e encontrou valor médio de gordura relativa de $24,1 \%$. Já no estudo conduzido por Velho (1994) com policiais militares do estado de Santa Catarina, na década de 90 , encontrou valores médios entre 15,9 e 18,1\%, dependendo do tempo de serviço dos militares.

Deve-se ressaltar que a comparação do \%G entre estudos apresenta limitações já que cada estudo citado utilizou equações de predição da densidade corporal diferentes. Para exemplificar esta limitação, Zorec (2001) analisou as características antropométricas de 172 policiais militares da Eslovênia, e estimou o \%G por meio de 3 diferentes equações (Lohman, Durnin e Womersley, Sloan e Weir). Os valores médios variaram de 9,4\% (Sloan e Weir), para 22,7\% (Durnin e Womersley) e $32,2 \%$ (Lohman), demonstrando uma alta variabilidade de valores dependendo das dobras utilizadas e da população a qual a equação de predição da densidade corporal foi validada.

\begin{tabular}{lcc}
$\mathrm{G} \%$ & $\mathrm{n}$ & $\%$ \\
\hline Normal & 01 & 2,0 \\
Acima do Normal & 08 & 15,7 \\
Tendência a Obesidade & 42 & 82,4 \\
Total & 51 & 100,0 \\
\hline \hline
\end{tabular}

Tabela 4. Distribuição dos policiais militares segundo a classificação da \% Gordura, de acordo com Pitanga (2008). Floriano-PI 
De A Tabela 5 apresenta a distribuição dos policiais militares de acordo com a Classificação do Nível de Atividade Física, apresentando-se que 50\% dos avaliados estão ativos sendo que a soma dos irregularmente ativos A e B totalizou $40 \%$ total da amostra. Em estudo realizado com o Comando de Policiamento da Capital (CPC) Recife-PE por Ferreira; Bonfim; Augusto, (2011) identificou que 72,6\% da amostra estudada ficou classificada como insuficientemente ativos, sendo este superior ao presente estudo.

Em estudo internacional o pesquisador Rodriguez - Añez (2003) fez uma junção de vários níveis de inatividade física, catalogando em seu estudo as pesquisas de outros autores. Assim, ele obteve os seguintes dados: os níveis de inatividade física no tempo de lazer a União Européia são de 26,9\% (MARTINEZ-GONZALEZ et al., 2001), dos Estados Unidos 29,9\% (PRATT, MACERA e BLANTON, 1999) e do Brasil são estimados em 60\% (FOLHA DE SÃO PAULO, 1997).

Sabendo-se que a inatividade trás riscos prejudiciais a saúde como obesidade, doenças coronárias, cardíacas e etc. O presente estudo nos traz uma preocupação muito grande, pois saber que nossos policiais que precisam sempre estar em forma para combater o crime e nos proteger estão com um nível de atividade tão insuficiente faz nos sentir desprotegidos.

\begin{tabular}{lcc}
\hline \hline \multicolumn{1}{c}{ Classificação do Nível de Atividade Física } & $\mathrm{n}$ & $\%$ \\
\hline Muito Ativo & 02 & 10 \\
Ativo & 10 & 50 \\
Irregularmente Ativo A & 03 & 15 \\
Irregularmente Ativo B & 05 & 25 \\
Sedentário & - & - \\
\hline \hline
\end{tabular}

Tabela 5. Distribuição dos policiais militares segundo a classificação do CELAFISCS e o Center for Disease Control (CDC) de Atlanta em 2002. 


\section{CONSIDERAÇÕES FINAIS}

De acordo com os resultados apresentados, pode-se concluir que, os policiais militares do $3^{\circ}$ Batalhão da cidade de Floriano estão fora da normalidade populacional no que se refere ao \%G, RCQ, IMC e inativos segundo o IPAQ, apresentando alto ou moderado risco a saúde.

É necessário urgentemente que novas políticas de qualidade de vida sejam adotadas na Corporação, visando à preservação do seu bem mais precioso que é o homem, sob pena de não cumprir de forma satisfatória a sua missão constitucional e ainda submeter todos os seus integrantes da atividade fim (área operacional) a contrair doenças que iram promover a sua aposentadoria precoce a diversos afastamentos para tratamentos médicos.

Portanto, tendo em vista os resultados encontrados neste estudo, recomendamse outras investigações, analisando a associação do estado nutricional, variáveis antropométricas e nível de atividade física em estudos com um número maior de policiais militares, além da realização de estudos longitudinais.

\section{REFERÊNCIAS BIBLIOGRAFICAS}

ARENA, Simone Sagres. Exercício físico e qualidade de vida: avaliação, prescrição e planejamento/Simone Sagres Arena. São Paulo: Phorte, 2009.240p. II.

CENTER DISEASE OF PREVENTION AND CONTROL.Promoting physical activity: a best buy in public health. 2000 .

FERREIRA, Daniela Karina da Silva; BONFIM, Cristine and AUGUSTO, Lia Giraldo da Silva. Fatores associados ao estilo de vida de policiais militares. Disponível em: Ciênc. saúde coletiva [online]. 2011, vol.16, n.8, pp. 3403-3412. ISSN 1413-8123. http://dx.doi.org/10.1590/S1413-81232011000900007. 
Folha de São Paulo. Caderno Mexa-se. São Paulo, 27 de novembro de 1997. p. 12. Pesquisa Data Folha.

FONTOURA, A.S. da; FORMENTIN, C. M. ;ABECH, E. A. ;Guia Prático de Avaliação Física: uma abordagem didática, abrangente e atualizada/ Andréa Silveira da Fontoura, Charles Marques Formentin, Everson Alves Abech.-São Paulo: Phorte, 2008.

GUEDES, D. P. Estudo da gordura corporal através da mensuração dos valores de densidade corporal e da espessura de dobras cutâneas em universitários.Santa Maria, 1985.Dissertação (MestradoemEducaçãoFísica) UFSM.

JACKSON, A. S.;POLLOCK, M. L.;WARD, A. Generalized equations for predicting body density of women. Medicine and Science in Sports and Exercise. V. 12, n. 3 , p. $175-182 ; 1980$.

JÁEQUIER, E. Energy, obesity and body weight Standars. Am. J. Clin. Nutr., [S.I.]; v. 45, p. 1.035-47, 1987.

LOPES, J.A. Prevalência de atividade física insuficiente e fatores associados em adultos no município de Lages, Santa Catarina: um estudo de base populacional. 66 páginas. Dissertação (Mestrado em Saúde Coletiva) Universidade do Planalto Catarinense - UNIPLAC. Orientadora: Profa. Dra. Marina Patrício de Arruda. Lages - SC, 2008.

LUZ, R. K.; LUCAS, R. D.; CAPUTO, F. Perfil antropométrico e somatotipico de policiais do bope do estado de santa catarina.Educação Física em Revista ISSN: 1983-6643 Vol.5 N³ set/out/nov/dez - 2011. 
MARTINS, C.O. Efeitos da ginástica laboral em servidores da reitoria da UFSC. Dissertação de mestrado na UFSC.Florianópolis 2000.

MARTINEZ-GONZALEZ, M. A.; VARO, J. J.; SANTOS, J. L.; DE IRALA, J.; GIBNEY, M.; KEARNEY, J. \& MARTINEZ, J. A. Prevalence of physical activity during leisure time in the Europe Union. Medicine and Science in Sports and Exercise, v. 33, p. 1142-1146. 2001.

MATSUDO, V.K.R.; MATSUDO, S.M.M. Atividade Física e esportiva na gravidez. In: TEDESCO, J.J.. A grávida. São Paulo: Athenu, 2000. P.59-81.

MINAYO, M. C. S.; ASSIS, S. G.; OLIVEIRA, R. V. C. Impacto das atividades profissionais na saúde física e mental dos policiais civis e militares do Rio de Janeiro (RJ, Brasil). Disponível em Ciênc. saúde coletiva [online]. 2011, vol.16, n.4, pp. 2199-2209. ISSN 1413-8123. http://dx.doi.org/10. 1590/S141381232011000400019.

NOBRE, G.C. (2008), “Análise dos componentes de aptidão física relacionada à saúde de bombeiros e policiais militares da região do Cariri Cearense, Brasil”. [Monografia apresentada ao curso de pós-graduação em fisiologia do exercício e treinamento desportivo] Juazeiro do Norte (CE), Universidade Castelo Branco.2008.

ORGANIZAÇÃO MUNDIAL DE SAÚDE - OMS. Physical status: the use and interpretation of anthropometry. Geneva: WHO, 1995. Disponível em: http:// www.unu.edu/unupress/food/FNBv27n4_suppl_2_final.pdf 
OMS. ORGANIZAÇÃO MUNDIAL DE SAÚDE. Obesity. Preventing and Managing the Global Epidemic. Report of a WHO Consultation on Obesity, Geneva: World Health Organization; 1997. p. 107-158.

OMS.ORGANIZAÇÃO MUNDIAL DA SAÚDE. Global Burden of Disease. Review. Genebra: Organização Mundial da Saúde. 2002

PETROSKI, E.L. “Antropometria: técnicas e padronizações”. Colaboradores Andiara Cleonice Schwingel et. al. Porto Alegre:Palotti. 1999.

PITANGA, F.J.G.Testes, medidas e avaliação em educação física e esportes I Francisco José GodimPitanga.- $5^{\circ}$ ed.- São Paulo: Phorte, 2008.

PORTO, Fausto Arantes. Benefícios da atividade física- em sua vida diária. Revista Saúde e movimento S/A, São Paulo, 2003.

PRATT, M.; MACERA, C. A.; BLANTON, C. Levels of physical activity in children and adults in United States: current evidence and research issues. MedSci. Sports Exerc., v.31, (Supl) p.526-533, 1999.

REIS JUNIOR, J..Avaliação da Composição Corporal em Policiais Militares do $22^{\circ}$ Batalhão da Polícia Militar do Estado de Goiás. Trindade-GO. Goiânia, 2009. Dissertação de Mestrado Universidade de Brasília.

RODRIGUEZ-Añez CR. Sistema de avaliação para a promoção e gestão do estilo de vida saudável e da aptidão física relacionada a saúde de policiais militares. [Tese de Doutorado-Doutorado em Engenharia de Produção]. Florianópolis-SC, Universidade Federal de Santa Catarina, 2003. 
SAVIO, K.E.O.; COSTA, T.H.M.; SCHMITZ, B.A.S.; SILVA, E.F. Sexo, renda e escolaridade associados ao nível de atividade física de trabalhadores. Disponível em Rev. Saúde Pública [online]. 2008, vol.42, n.3, pp. 457-463. EpubApr 10, 2008. ISSN 0034-8910.http://dx.doi.org/10. 1590/S0034-89102008005000018.

VELHO, M.N. (1994), "Análise da aptidão física dos policiais militares do Estado de Santa Catarina". [Dissertação de Mestrado em Educação Física - Programa de Pós-Graduação em Ciência do Movimento Humano] Santa Maria (RS), Universidade Federal de Santa Maria. 1994.

ZOREC B. "Anthropometric Characteristics in Police Officers". Journal of Criminal Justice and Security.;v.1. p. 26-35, 2001. 


\title{
PHYSICAL ACTIVITY LEVEL AND NUTRITIONAL STATUS OF MILITARY POLICE IN FLORIANO-PI CITY.
}

\begin{abstract}
The objective was to assess the level of physical activity and nutritional status of the military police of the city of Floriano, Piauí. The survey was conducted in the 3rd Battalion of Military Police of the city of Floriano, always at 6: 30h am to anthropometric assessments and evaluation of the physical activity level used the IPAQ. According to the results presented, it can be concluded that the military police of the 3rd Battalion of the city of Floriano are outside the normal population with regard to \% G, WHR, BMI and inactive according to the IPAQ, with high or moderate risk to health.
\end{abstract}

Key Words: Nutritional Status. Physical activity level. Military Police.

\section{NIVEL DE ACTIVIDAD FÍSICA Y EL ESTADO NUTRICIONAL POLICÍA MILITAR CIUDAD FLORIANO-PI.}

Resumen: El objetivo fue evaluar el nivel de actividad física y el estado nutricional de la policía militar de la ciudad de Floriano, Piauí. La encuesta se realizó en el 3er Batallón de Policía Militar de la ciudad de Floriano, siempre a las 6: 30h de la mañana a las evaluaciones y evaluación del nivel de actividad física antropométricas utilizó el IPAQ. De acuerdo con los resultados presentados, se puede concluir que la policía militar del 3er Batallón de la ciudad de Floriano están fuera de la población normal con respecto al\% G, RHO, IMC e inactivos de acuerdo con el IPAQ, con alta o riesgo moderado para la salud.

Palabras clave: Estado Nutricional. Nivel de Actividad Física. Policia Militar. 